\title{
Solving Large Batches of Traveling Salesman Problems with Parallel and Distributed Computing
}

\author{
S. G. Ozden ${ }^{\mathrm{a}}$, A. E. Smith ${ }^{\mathrm{a}, *}$, K.R. Gue ${ }^{\mathrm{b}}$ \\ ${ }^{a}$ Department of Industrial $\mathcal{E}$ Systems Engineering, Auburn University, Auburn, AL 36849, USA \\ ${ }^{b}$ Department of Industrial Engineering, University of Louisville, Louisville, KY 40292, USA
}

\begin{abstract}
In this paper, we describe and compare serial, parallel, and distributed solver implementations for large batches of Traveling Salesman Problems using the Lin-Kernighan Heuristic (LKH) and the Concorde exact TSP Solver. Parallel and distributed solver implementations are useful when many medium to large size TSP instances must be solved simultaneously. These implementations are found to be straightforward and highly efficient compared to serial implementations. Our results indicate that parallel computing using hyper-threading for solving 150- and 200-city TSPs can increase the overall utilization of computer resources up to 25 percent compared to single thread computing. The resulting speed-up/physical core ratios are as much as ten times better than a parallel and concurrent version of the LKH heuristic using $\mathrm{SPC}^{3}$ in the literature. For variable TSP sizes, a longest processing time first heuristic performs better than an equal distribution rule. We illustrate our approach with an application in the design of order picking warehouses.
\end{abstract}

Keywords: TSP, Parallel Computing, Routing, Distributed Computing, Facilities Design

\section{Introduction}

With the arrival of multi-core processors in 2005, computers gained more power by providing more clock cycles per CPU. However, most software implementations are still inefficient single processor programs [1]. Writing an efficient and scalable parallel program is a complex task. However, C\# parallel libraries provide the power of parallel computing with simple changes in the implementation if a certain condition is met: the steps inside the operation must be independent (i.e., they must not write to memory locations or files that are accessed by other steps). Solving large batches of Traveling Salesman Problems is an example of such independent operations. Each TSP instance can be solved by calling a TSP Solver in parallel. Applications of large batches of TSPs include design of order picking warehouses [2], large

\footnotetext{
${ }^{*}$ Corresponding author

Email addresses: gokhan@auburn.edu (S. G. Ozden), smithae@auburn.edu (A. E. Smith ), kevin.gue@louisville.edu (K.R. Gue)
} 
scale distribution network simulation [3, 4], case-based reasoning for repetitive TSPs [5], and delivery route optimization [6]. In these applications the TSP solving consumes most of the computational effort.

We use both the Lin-Kernighan Heuristic (LKH) and the Concorde exact TSP Solver (Concorde). The methods we describe are applicable to optimization problems that must be solved repetitively in an overall algorithm. In this paper, we present two example problems that solve large batches of TSPs and give implementation details in the context of warehouse design for order picking operations. The main result of this paper is to show that doing the parallelism at the TSP level instead of the TSP Solvers' implementation level [1] provides better CPU utilization. A parallel implementation generally achieves better CPU execution times than serial implementations, but an improved CPU utilization is not easily achievable. To the best of our knowledge, this is the first work that presents CPU utilizations for solving large batches of TSPs in serial, parallel, and distributed computing environments.

This work is organized as follows. In Section 2 we give a technical description of the Traveling Salesman Problem (TSP) with solution techniques and its variant of large batches of Traveling Salesman Problems. In Section 3, we describe our implementation of serial, parallel, and distributed large batches of TSPs solvers. In Section 4 we present the computational results, and in Section 5 we offer conclusions.

\section{Traveling Salesman Problem and Solution Techniques}

The Traveling Salesman Problem (TSP) is an NP-hard [7] combinatorial optimization problem where a salesman has to visit $n$ cities only once and then return to the starting city with minimum travel cost (or travel distance). It is one the most famous and widely studied combinatorial problems [8]. Solving the problem with a brute force approach requires a factorial execution time $O(n !)$ by permuting all the possible tours through $n$ cities and therefore checking $(n-1)$ ! possible tours. Given a starting city, there can be $n-1$ choices for the second city, $n-2$ choices for the third city, and so on. In the symmetric TSP, the number of possible solutions is halved because every sequence has the same distance when traveled in reverse order. If $n$ is only 20 , there are approximately $10^{18}$ possible tours. In the asymmetric TSP, costs on an arc might depend on the direction of travel (streets might be one way or traffic might be considered). 
Using an integer linear programming formulation [1], the TSP can be defined as:

$$
\begin{gathered}
\min \sum_{i \in V} \sum_{j \in V} c_{i j} x_{i j} \\
\sum_{j \in V} x_{i j}=1, i \in V \\
\sum_{i \in V} x_{i j}=1, j \in V \\
\sum_{i \in S} \sum_{j \in S} x_{i j} \leq|S|-1, \forall S \subset V, S \neq \emptyset \\
x_{i j} \in\{0,1\}, \forall i, j \in V
\end{gathered}
$$

where $x_{i j}=1$ if the path goes from city $i$ to city $j$ and 0 otherwise. $V$ is a set of cities, $S$ is a subset of $V$, and $c_{i j}$ is the cost of moving from city $i$ to city $j$. The first set of equalities enforces that each city be arrived at from exactly one city, and the second set enforces that from each city there is a departure to exactly one other city. The third set of constraints ensures that a single tour is created which spans all cities.

TSP is a widely studied problem where solution methods can be classified as Exact Algorithms, TSP Heuristics, or Meta-Heuristics. Exact algorithms are guaranteed to find an optimal solution in a bounded number of steps. Enumeration is only good for solving small instances up to 10 cities. The dynamic programming algorithm of Held and Karp [9] and the branch-and-bound algorithm are some well known algorithms in this class. They are good for solving instances up to 60 cities. Concorde is a code for solving symmetric TSPs and related network optimization problems exactly using branch-and-bound and problem specific branch-and-cut techniques [10,11]. This algorithm is the current exact method of choice for solving large instances. Concorde was able to solve a 85,900-city problem in TSPLIB [12].

Heuristics are used when the problem is large and a solution is needed in a limited amount of time. We can categorize these heuristics into two groups: "constructive heuristics" and "improvement heuristics." Constructive heuristics start with an empty tour and repeatedly extend the tour until it is complete. The most popular constructive heuristic is the nearest neighbor algorithm, where the salesman chooses the nearest unvisited city as the next move and finally returns to the first city. Improvement heuristics start with a complete tour and then try to improve it with local moves. The most popular and easily implementable heuristic is the pairwise exchange, or 2-opt, which iteratively removes two edges and replaces them with two different edges to obtain a shorter tour. The algorithm continues until no more improvement is possible. $k$-opt is a generalization which forms the basis of one of the most effective heuristics for solving the symmetric TSP, the Lin-Kernighan Heuristic [13]. $k$-opt is based on the concept 
of k-optimality: "A tour is said to be k-optimal if it is impossible to obtain a shorter tour by replacing any $k$ of its links by any other set of $k$ links" [14]. For a more detailed review of these algorithms refer to $[14]$.

Meta-heuristic algorithms are designed for solving a problem more quickly than exact algorithms but are not specifically designed for any particular problem class. Most of these meta-heuristics implement some form of stochastic optimization. The solution is dependent on the set of random numbers generated. Meta-heuristics' ability to find their way out of local optima contributes to their current popularity. Specific meta-heuristics used for solving the TSP include simulated annealing [15], genetic algorithms [16], tabu search [17], ant colony optimization [18], iterated local search [19], particle swarm optimization [20], nested partitions [21], and neural networks [22]. There are many variants and hybrids of these metaheuristics designed to solve the TSP [23].

\subsection{Parallel/Distributed Implementations}

The algorithms mentioned in this section solve a single TSP using parallel/distributed techniques. A parallel and concurrent version of the Lin-Kernighan-Helsgaun heuristic using $\mathrm{SPC}^{3}$ programming is implemented in [1]. $\mathrm{SPC}^{3}$ is a newly developed parallel programming model (Serial, Parallel, and Concurrent Core to Core Programming Model) developed for multi-core processors. Developers can easily write new parallel code or convert existing code written for a single processor. All of their speedups were less than 2 times compared to single thread runs, even when using a 24 -core machine. The computational time of each individual task parallelized was insignificantly small, therefore the overhead of the parallelization prevented achievement close to the theoretical boundaries of the speed-up [24]. In [25], a sequential algorithm is developed for solving TSP and converted into a parallel algorithm by integrating it with the Message Passing Interface (MPI) libraries. The authors use a dynamic two dimensional array and store the costs of all possible paths. They decompose the task of filling this 2D array into subroutines to parallelize the algorithm using MPI. The Message Passing Interface provides the subroutines needed to decompose the tasks involved in the TSP solving process into subproblems that can be distributed among the available nodes for processing. Experimental results conducted on a Beowulf cluster show that their speed-ups were less than 3.5 times on a 32 processor cluster. Another technique to implement parallel heuristics for the geometric TSP (symmetric and Euclidean distances between cities), called the divide and conquer strategy, is proposed in [26]. This reference subdivides the set of cities into smaller sets and computes an optimal subtour for each subset. Each subtour is then combined to obtain the tour for the entire problem. The author was able to achieve between 3.0 and 7.2 times speed-up on a 16 core machine. 


\subsection{Large Batches of Traveling Salesman Problems}

Solving a single TSP gives the best path for a certain instance. However, this assumes that the location of the cities (visited points) are fixed. In situations where the problem consists of finding the optimal locations of these cities (visited points), numerous TSPs must be solved to assess a certain design, (e.g, a warehouse layout or a distribution network). Large batches of TSPs are different from the multiple traveling salesman problem (mTSP) which consists of determining a set of routes for $m$ salesmen who all start from and return back to a depot. In large batches of TSPs, to find the expected distance traveled (or another relevant statistic of the distribution of tour lengths), we need to evaluate a large set of tours. Solving large batches of TSPs provides a more accurate estimate and a wider information set than solving only a single TSP. This type of problem can be found in design of order picking warehouses, large scale distribution network simulation [3, 4], case-based reasoning for repetitive TSPs [5], and delivery route optimization [6]. Since our focus is solving large batches of TSPs, parallelizing each task at the TSP level will lead to better speed-ups than solving a single TSP using parallel techniques. To best of our knowledge, ours is the first comprehensive comparison of serial, parallel, and distributed large batches of TSPs solvers.

\section{Methodology}

The methodology for solving large batches of TSPs includes three main steps. In Step 1, problem specific conditions are set (e.g., a warehouse layout structure or a distribution network structure). This typically involves creating locations and calculating distances between locations. We need to assess the total or average cost of TSPs for the given configuration of Step 1. Therefore, multiple TSPs are created and evaluated with a TSP solver in Step 2. In Step 3, the total or average cost of the TSPs is calculated. In the next subsection, we provide an example from the literature in the context of a large-scale distribution network simulation [3]. In the later subsections, we discuss this problem in the context of design of order picking warehouses and give implementation details.

\subsection{Large-scale Distribution Network Simulation}

A large scale distribution network may look like in Figure 1. This network includes multiple manufacturing enterprises. Parts are delivered from suppliers directly to factories or through warehouses. Direct delivery from the supplier to a warehouse or a factory would be inefficient. Therefore, a truck visits several suppliers and collects parts.

According to [3], the total cost of distribution must be calculated. Therefore, several hundreds of distributing routes are created for differing conditions to find the best large-scale distribution network. 


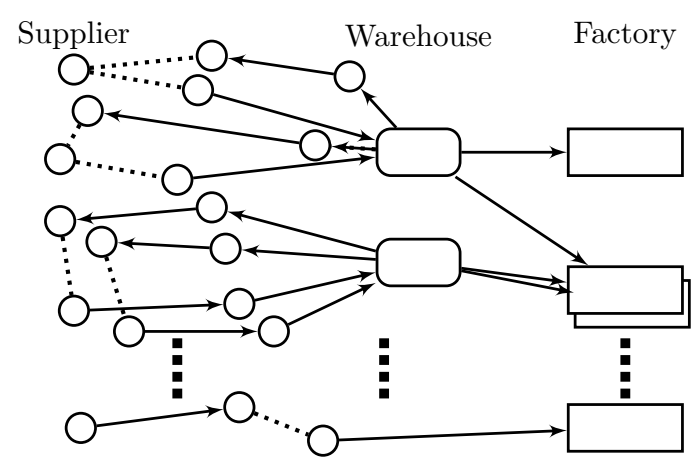

Figure 1: Large-scale Distribution Network [3]

The authors in [3] note that efficiency (i.e., solving overall problem quickly) and precision (i.e., solving close to optimal) are important. The methodology herein is applicable to this class of distribution network problem.

\subsection{Design of Order Picking Warehouses}

The warehouse design software that motivated this study creates a warehouse layout for given parameters, calculates shortest path distances between storage locations and between storage locations to a pick and deposit location (i.e., a location where a TSP tour starts and ends), creates a TSP file based on the product orders (i.e., each order is a pick tour or a TSP), sends them to the Concorde or LKH solver and reads the resulting TSP distance from these solvers [2]. To find the best designs, we must consider numerous product orders that represent the order picking activity of the warehouse. We identify the design that gives the minimum average travel cost.

Figure 2 shows the seven stages in the warehouse layout creation and fitness assessment. Boxes with dashed lines represent the most time consuming parts of the overall process. In this paper, we focus on Stage 6: "Create a TSP File for Each Order and Solve TSPs." We will describe how we implemented parallel and distributed computing techniques for this stage. Our approach is not specific to warehouse design and can be used for any application that requires solving large batches of TSPs.

We use the C\# programming language and the .NET environment. We use C\# parallel class methods [27] for parallel computing. For distributed computing, we implement a modified version of the asynchronous client-server example from the Microsoft Developer Network (MSDN) [28].

\subsection{Serial Execution of Concorde/LKH}

In this case, we send a set of orders (pick lists) to a wrapper function one by one in serial. For each order, we find the products and their locations in the warehouse and generate a distance matrix that contains only the items in this particular order. Because the shortest path distances are already 


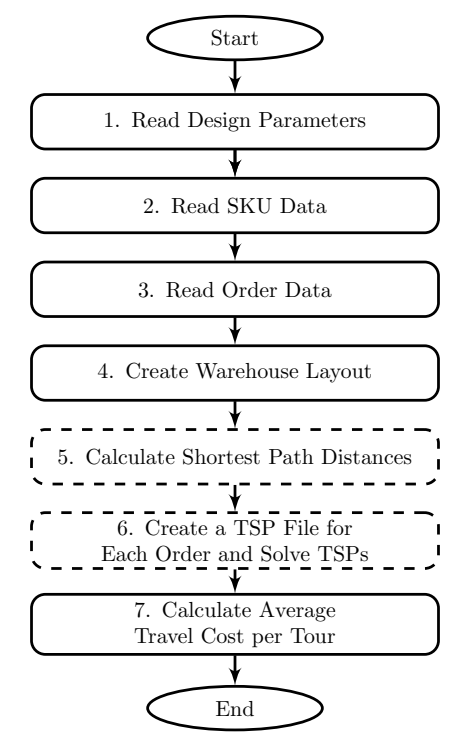

Figure 2: Stages in warehouse design creation and fitness assessment

calculated in the previous stage, we only generate a sub-matrix of the main distance matrix which contains the all-pairs shortest path distances between every storage location and the pick and deposit location in the warehouse. Based on this distance matrix, we generate a file in the TSP file format [12]. Concorde or LKH is called to solve the corresponding TSP file, and to read and keep the distance after the execution. Finally, we delete the generated TSP file and any generated files from Concorde/LKH and continue to the next order. Stage 6 ends when all orders are evaluated.

\subsection{Parallel Execution of Multiple Concorde/LKH Solvers}

Since the operation inside the loop of Figure 3 is independent of any other, we can use "Parallel For Loop" [24] and send a set of orders to the wrapper function in parallel. The rest of the operations are the same as a serial execution, but they are performed in parallel until all orders are completed. In Figure 4, blocks with dashed lines represent the operations that are performed concurrently.

\subsection{Parallel and Distributed Execution of Multiple Concorde/LKH Solvers}

In this case, we have a master-slave architecture to perform distributed computing. We use a static load balancing methodology to distribute TSPs evenly among machines because dynamic load balancing methodologies increase the network overhead by sending and receiving the status of each processor of each slave machine. Also in our first set of experiments, we analyze TSPs of the same size in one batch, which makes dynamic load balancing less effective. We first create TSP files of each order in the master machine in parallel and distribute the TSP files to each slave machine using the TCP/IP protocol with given workload percentage. If the master machine requires provably optimal solutions, then it sends the 


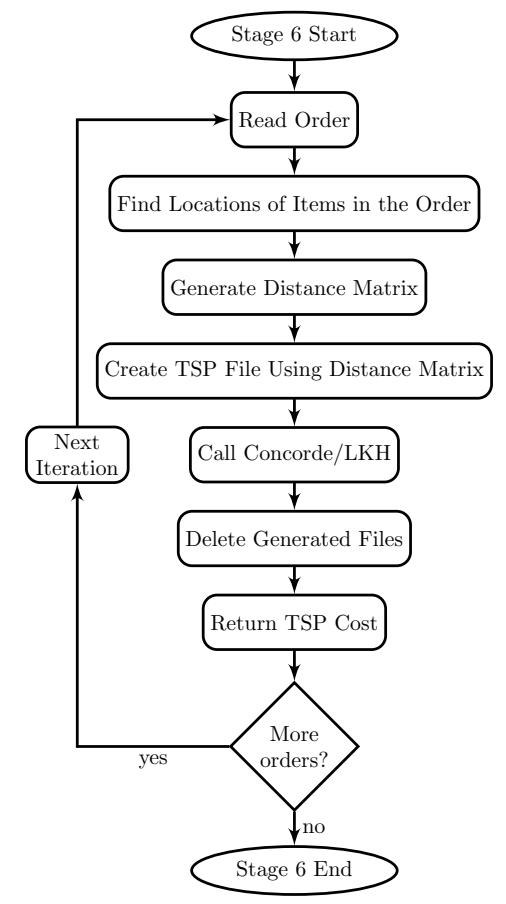

Figure 3: Serial Execution of Concorde/LKH Solvers

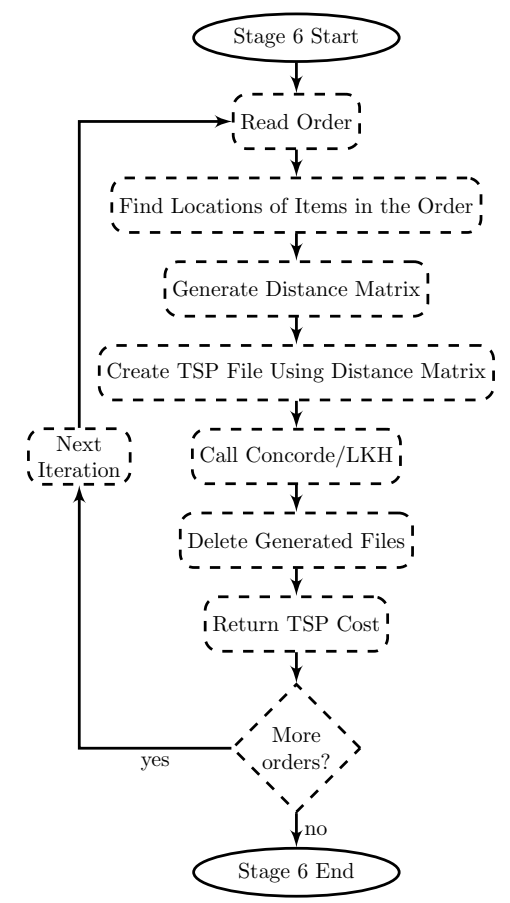

Figure 4: Parallel Execution of Concorde/LKH Solvers 


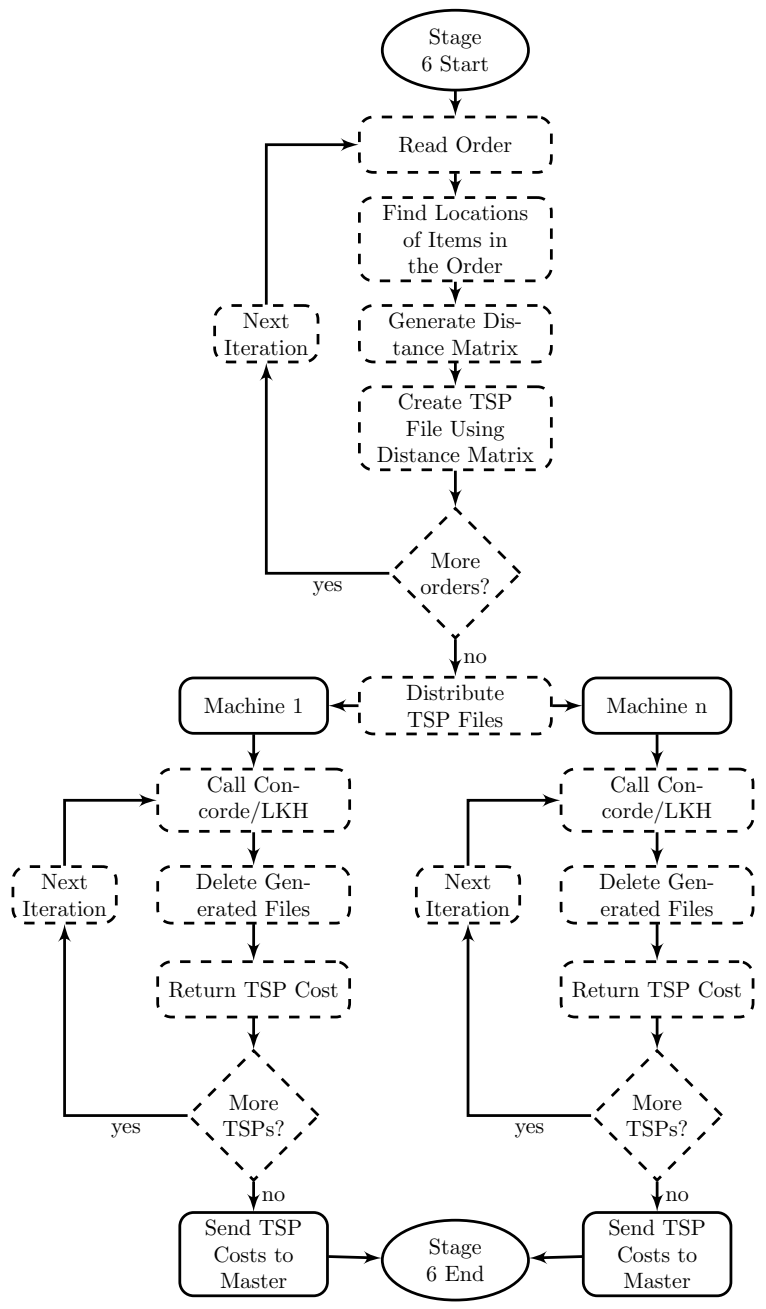

Figure 5: Parallel and Distributed Execution of Multiple Concorde/LKH Solvers

TSP files using port 8888 otherwise it uses port 8889 . Two processes are running on the slave machine. The first listens to port 8888 and solves the TSP files that are sent by the master machine with Concorde in parallel. The second listens to port 8889 and solves the TSP files that are sent by the master machine with the LKH in parallel. A slave machine receives the TSP files over the TCP/IP protocol and keeps the files until Stage 6 ends. The slave machine waits for a "DONE" signal to start TSP runs in parallel, then returns the TSP distance over TCP/IP with the "DONE" signal at the end, closes the communication between the master and slave machines. After the master machine receives all TSP distances, Stage 6 ends. Figure 5 shows the parallel and distributed execution of multiple Concorde/LKH solvers. Blocks with dashed lines represent the operations that are performed concurrently. 
Table 1: Problem parameters used for the computational experiments

\begin{tabular}{|l|l|}
\hline Number of Cities & $5,25,50,100,150,200$ \\
\hline Number of TSPs Solved & $10,100,1000,10000$ \\
\hline Execution Mode & Serial, Parallel, Distributed with two or three Machines \\
\hline
\end{tabular}

\section{Computational Results}

\subsection{Fixed Size TSP Instances}

We have selected fixed sized TSP instances generated using our warehouse optimization software. For the execution of the algorithms, a Lenovo workstation with a six core hyperthreaded Intel Xeon E5-1650 processor is used. Each workstation has 64GB of RAM and 256GB of Solid State Drive. The operating system is 64 bit Windows 7, Enterprise Edition. The total number of parallel threads that can be executed is $2 \times 6=12$. Table 1 gives a summary of the parameter settings used in the set of experiments. To address the variability in execution times as a result of the background processes of the operating system, we perform five runs for each experiment and calculate average execution time. We use default settings for Concorde. For LKH, we set "RUNS" (the total number of runs) to 1, "TIME_LIMIT" to 1 second and "MOVE_TYPE" to 4 which means that a sequential 4-opt move is to be used.

Table 2 shows the average execution time speed up over serial execution. Speed up is the ratio of the execution time of the serial algorithm to the execution time of the parallel/distributed algorithm. Both LKH and Concorde better utilize computing resources when the TSP size increases (see Figures 6 and 7). LKH uses parallel executions better than Concorde, because it is set to run with a maximum execution time for each TSP instance. As a result, all concurrent operations have similar execution times, which enables a better workload balance among CPU cores. When solving with Concorde, some TSP instances are harder, therefore the time to find an optimal solution varies, leading to a poor workload balance among cores (and CPUs for distributed computing).

However, Concorde uses distributed-2 and distributed-3 executions more efficiently because the overhead of sending TSP files to slaves has less effect in execution of Concorde than LKH (see Figures 8 and 9). In Figure 6, the speed-up decreases when solving for instances with fewer than 50 cities and more than 100 TSPs. This is because the network overhead of sending many TSP files becomes inefficient for few cities with Concorde. The same is true for LKH, but in this case the speed-up decreases even for a 50 city TSP. There is a point between the number of TSPs (many) and the city size (low) for both TSP Solvers where distributed computing becomes inefficient.

Table 3 shows the average speed-up/physical core ratio (SPR) of the additional CPU cores for parallel, distributed-2, and distributed-3 executions. It is important to note that serial execution uses one of the six physical cores instead of one of the twelve logical cores. A hyperthreaded processor can achieve 30 
Table 2: Average Speed-ups (Multiplier)

\begin{tabular}{|c|c|c|c|c|c|c|c|}
\hline \multirow[b]{2}{*}{ Cities } & \multirow[b]{2}{*}{ Batch } & \multicolumn{2}{|c|}{ Parallel } & \multicolumn{2}{|c|}{ Distributed 2} & \multicolumn{2}{|c|}{ Distributed 3} \\
\hline & & Concorde & LKH & Concorde & LKH & Concorde & LKH \\
\hline \multirow[t]{4}{*}{5} & 10 & 3.68 & 3.13 & 5.87 & 4.30 & 7.17 & 5.80 \\
\hline & 100 & 4.63 & 3.77 & 7.82 & 5.16 & 10.31 & 6.96 \\
\hline & 1000 & 3.42 & 4.25 & 6.28 & 6.30 & 8.01 & 7.89 \\
\hline & 10000 & 3.34 & 4.03 & 5.31 & 4.68 & 6.39 & 5.48 \\
\hline \multirow[t]{4}{*}{25} & 10 & 4.69 & 3.95 & 6.28 & 4.40 & 6.31 & 4.87 \\
\hline & 100 & 4.54 & 4.44 & 9.87 & 6.09 & 12.69 & 7.40 \\
\hline & 1000 & 3.73 & 5.63 & 10.35 & 7.56 & 13.58 & 9.29 \\
\hline & 10000 & 3.73 & 5.16 & 9.22 & 5.87 & 12.76 & 7.70 \\
\hline \multirow[t]{4}{*}{50} & 10 & 3.79 & 4.01 & 4.78 & 4.64 & 4.12 & 5.35 \\
\hline & 100 & 3.73 & 6.14 & 7.09 & 8.10 & 6.39 & 9.73 \\
\hline & 1000 & 4.65 & 6.75 & 10.03 & 9.19 & 13.70 & 12.25 \\
\hline & 10000 & 4.58 & 6.80 & 10.52 & 8.85 & 15.02 & 11.57 \\
\hline \multirow[t]{4}{*}{100} & 10 & 4.62 & 4.13 & 4.70 & 4.47 & 5.25 & 4.66 \\
\hline & 100 & 5.44 & 6.71 & 8.06 & 10.48 & 10.15 & 12.95 \\
\hline & 1000 & 6.62 & 6.98 & 12.03 & 11.34 & 16.87 & 15.90 \\
\hline & 10000 & 6.80 & 7.22 & 13.21 & 12.08 & 19.35 & 16.74 \\
\hline \multirow[t]{4}{*}{150} & 10 & 1.46 & 4.16 & 1.25 & 3.98 & 1.36 & 4.34 \\
\hline & 100 & 5.51 & 6.58 & 5.87 & 9.46 & 5.60 & 11.81 \\
\hline & 1000 & 6.69 & 7.07 & 11.06 & 10.80 & 14.51 & 14.33 \\
\hline & 10000 & 7.20 & 7.37 & 12.53 & 11.50 & 17.76 & 14.79 \\
\hline \multirow[t]{4}{*}{200} & 10 & 2.47 & 3.64 & 1.45 & 3.47 & 2.83 & 3.59 \\
\hline & 100 & 5.25 & 6.80 & 5.20 & 7.72 & 5.95 & 9.37 \\
\hline & 1000 & 6.49 & 7.13 & 10.60 & 9.28 & 12.51 & 11.86 \\
\hline & 10000 & 6.07 & 7.49 & 12.30 & 10.13 & 15.01 & 12.92 \\
\hline
\end{tabular}




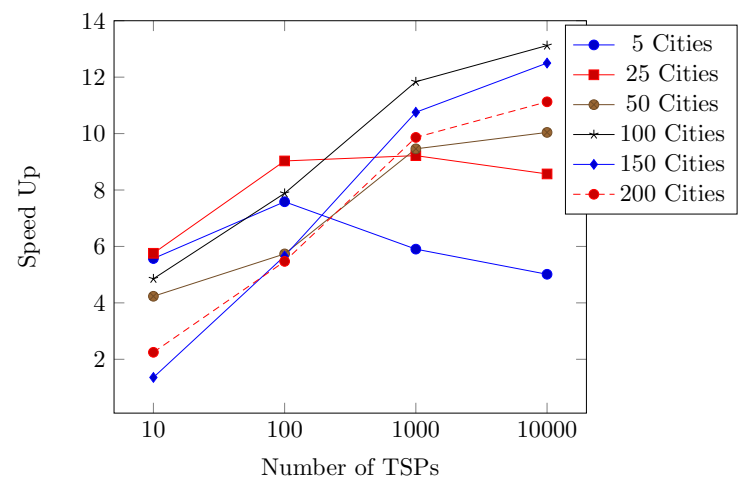

Figure 6: Number of TSPs vs. Number of Cities for Speed Up (Concorde)

percent increased performance compared to a non-hyperthreaded processor [29]. Therefore we should be able to see SPR values as high as $130 \%$. Values less then $100 \%$ mean that executions with parallel or distributed techniques do not effectively use the physical cores. Values higher than $100 \%$ mean that executions with parallel or distributed techniques use computing resources more effectively than serial execution because of hyper-threading. This means that parallelization done at a higher level (solving each entire TSP in parallel) improves CPU utilization over parallelization done at Stage 6 (finding an optimal tour by performing a number of trials where each trial attempts to improve the initial tour) for LKH using SPC $^{3}[1]$. Moreover, a parallel implementation can solve more than six times faster than a serial implementation on the same 6-core machine. We used the following formula to calculate the SPR values in Table 3 :

$$
s p r=\frac{s u}{n p c}
$$

where $s p r$ is the speed-up/physical core ratio, su is the average execution time speed-up over serial execution, and $n p c$ is the number of physical cores available per execution. $n p c$ values for parallel, distributed-2, and distributed-3 executions are 6, 12, and 18, respectively. Table 4 shows a 4 -way ANOVA using Minitab 17.0 statistical software. The differences between the group means of the main effects (TSP size, number of TSPs (batch), algorithm type (parallel, distributed-2, and distributed-3), solver type (LKH or Concorde)) and their interactions (two, three, and four level interactions) are all statistically significant. The model can explain $94.72 \%$ of the variability of the response data around its mean.

\subsection{Variable Size TSP Instances}

We should emphasize that our methodology is not bounded to fixed size TSP instances. In this set of experiments, we demonstrate our methodology's ability to solve variable size TSP instances. These instances are from real order picking data where TSP sizes are different. This real order data set has 
Table 3: Average Speed-up/Physical Core Ratio (Percent) and 95\% Confidence Intervals on SPR

\begin{tabular}{|c|c|c|c|c|c|c|c|}
\hline & & \multicolumn{2}{|c|}{ Parallel } & \multicolumn{2}{|c|}{ Distributed 2} & \multicolumn{2}{|c|}{ Distributed 3} \\
\hline Cities & Batch & Concorde & LKH & Concorde & LKH & Concorde & LKH \\
\hline \multirow[t]{4}{*}{5} & 10 & $61(52,70)$ & $52(41,63)$ & $49(41,56)$ & $36(25,47)$ & $40(27,53)$ & $32(22,43)$ \\
\hline & 100 & $77(75,79)$ & $63(59,66)$ & $65(62,68)$ & $43(42,44)$ & $57(55,60)$ & $39(37,41)$ \\
\hline & 1000 & $57(57,57)$ & $71(70,72)$ & $52(51,54)$ & $52(52,53)$ & $45(43,46)$ & $44(43,44)$ \\
\hline & 10000 & $56(55,56)$ & $67(67,68)$ & $44(44,45)$ & $39(38,40)$ & $36(35,36)$ & $30(30,31)$ \\
\hline \multirow[t]{4}{*}{25} & 10 & $78(73,83)$ & $66(58,74)$ & $52(46,58)$ & $37(36,38)$ & $35(30,40)$ & $27(21,33)$ \\
\hline & 100 & $76(72,79)$ & $74(71,77)$ & $82(80,85)$ & $51(48,54)$ & $70(67,74)$ & $41(40,42)$ \\
\hline & 1000 & $62(60,64)$ & $94(89,99)$ & $86(85,87)$ & $63(61,65)$ & $75(72,79)$ & $52(50,53)$ \\
\hline & 10000 & $62(62,63)$ & $86(85,87)$ & $77(76,77)$ & $49(48,50)$ & $71(70,72)$ & $43(41,44)$ \\
\hline \multirow[t]{4}{*}{50} & 10 & $63(52,74)$ & $67(64,70)$ & $40(36,44)$ & $39(36,41)$ & $23(21,25)$ & $30(28,32)$ \\
\hline & 100 & $62(54,71)$ & $102(98,106)$ & $59(55,64)$ & $67(66,69)$ & $35(30,41)$ & $54(51,57)$ \\
\hline & 1000 & $77(72,83)$ & $113(111,114)$ & $84(79,88)$ & $77(76,77)$ & $76(67,86)$ & $68(67,69)$ \\
\hline & 10000 & $76(76,77)$ & $113(112,115)$ & $88(85,90)$ & $74(73,74)$ & $83(82,85)$ & $64(63,66)$ \\
\hline \multirow[t]{4}{*}{100} & 10 & $77(46,108)$ & $69(64,73)$ & $39(33,45)$ & $37(36,39)$ & $29(23,35)$ & $26(26,26)$ \\
\hline & 100 & $91(78,103)$ & $112(107,117)$ & $67(55,80)$ & $87(82,92)$ & $56(48,65)$ & $72(66,78)$ \\
\hline & 1000 & $110(108,113)$ & $116(114,118)$ & $100(89,111)$ & $95(93,96)$ & $94(90,98)$ & $88(86,90)$ \\
\hline & 10000 & $113(113,114)$ & $120(119,122)$ & $110(109,112)$ & $101(98,103)$ & 107(104,110) & $93(92,94)$ \\
\hline \multirow[t]{4}{*}{150} & 10 & $24(22,27)$ & $69(61,78)$ & $10(5,15)$ & $33(30,37)$ & $8(6,9)$ & $24(24,25)$ \\
\hline & 100 & $92(79,104)$ & $110(102,117)$ & $49(39,59)$ & $79(75,83)$ & $31(21,41)$ & $66(61,70)$ \\
\hline & 1000 & $112(110,113)$ & $118(116,120)$ & $92(88,97)$ & $90(88,92)$ & $81(74,87)$ & $80(75,84)$ \\
\hline & 10000 & $120(118,122)$ & $123(122,124)$ & $104(99,110)$ & $96(95,97)$ & $99(92,105)$ & $82(81,84)$ \\
\hline \multirow[t]{4}{*}{200} & 10 & $41(25,57)$ & $61(58,63)$ & $12(6,18)$ & $29(28,30)$ & $16(8,23)$ & $20(19,21)$ \\
\hline & 100 & $88(76,100)$ & $113(111,116)$ & $43(22,65)$ & $64(59,69)$ & $33(22,44)$ & $52(46,58)$ \\
\hline & 1000 & $108(103,114)$ & $119(116,121)$ & $88(82,94)$ & $77(75,80)$ & $69(46,93)$ & $66(63,68)$ \\
\hline & 10000 & $101(90,113)$ & $125(124,125)$ & $103(96,109)$ & $84(84,85)$ & $83(67,100)$ & $72(71,73)$ \\
\hline
\end{tabular}

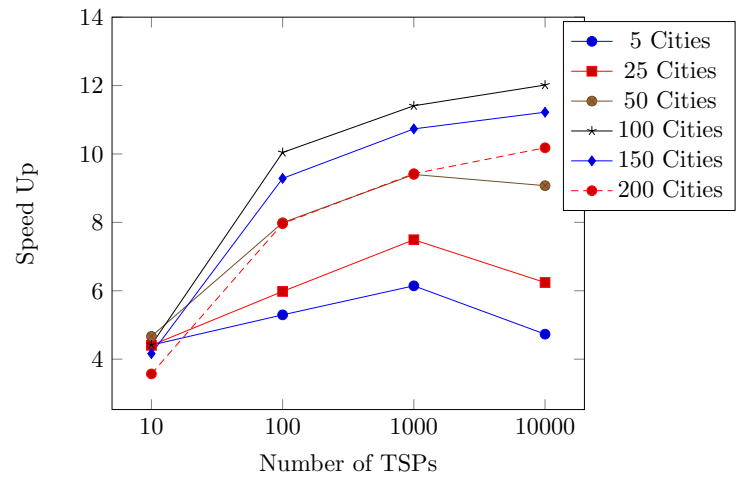

Figure 7: Number of TSPs vs. Number of Cities for Speed Up (LKH) 
Table 4: ANOVA: SPR versus Size, Batch, Algorithm, Solver

\begin{tabular}{l|r|r|r|r|r}
\hline Source & DF & Adj SS & Adj MS & F-Value & P-Value \\
\hline Size & 5 & 7.6132 & 1.52264 & 275.88 & 0 \\
Batch & 3 & 21.4787 & 7.15956 & 1297.22 & 0 \\
Algorithm & 2 & 12.0637 & 6.03184 & 1092.89 & 0 \\
Solver & 1 & 0.078 & 0.078 & 14.13 & 0 \\
Size*Batch & 15 & 7.7685 & 0.5179 & 93.84 & 0 \\
Size*Algorithm & 10 & 0.7865 & 0.07865 & 14.25 & 0 \\
Size*Solver & 5 & 1.3363 & 0.26725 & 48.42 & 0 \\
Batch*Algorithm & 6 & 0.4232 & 0.07054 & 12.78 & 0 \\
Batch*Solver & 3 & 0.3713 & 0.12377 & 22.42 & 0 \\
Algorithm*Solver & 2 & 1.2914 & 0.64569 & 116.99 & 0 \\
Size*Batch*Algorithm & 30 & 0.7685 & 0.02562 & 4.64 & 0 \\
Size*Batch*Solver & 15 & 1.524 & 0.1016 & 18.41 & 0 \\
Size*Algorithm*Solver & 10 & 0.4784 & 0.04784 & 8.67 & 0 \\
Batch*Algorithm*Solver & 6 & 0.5061 & 0.08435 & 15.28 & 0 \\
Size*Batch*Algorithm*Solver & 30 & 0.4957 & 0.01652 & 2.99 & 0 \\
\hline Error & 576 & 3.179 & 0.00552 & & \\
\hline Total & 719 & 60.1625 & & & \\
\hline & S & R-sq & R-sq(adj) & R-sq(pred) & \\
\hline & 0.0742909 & $94.72 \%$ & $93.40 \%$ & $91.74 \%$ & \\
\hline
\end{tabular}

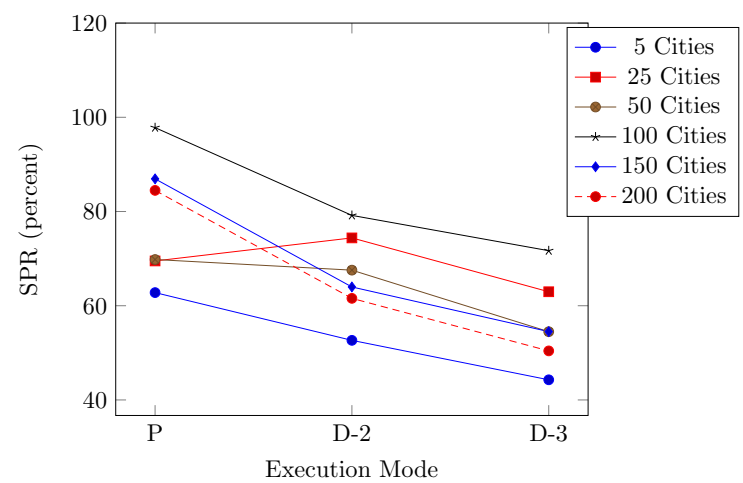

Figure 8: Execution Mode vs. Number of Cities for SPR (Concorde)

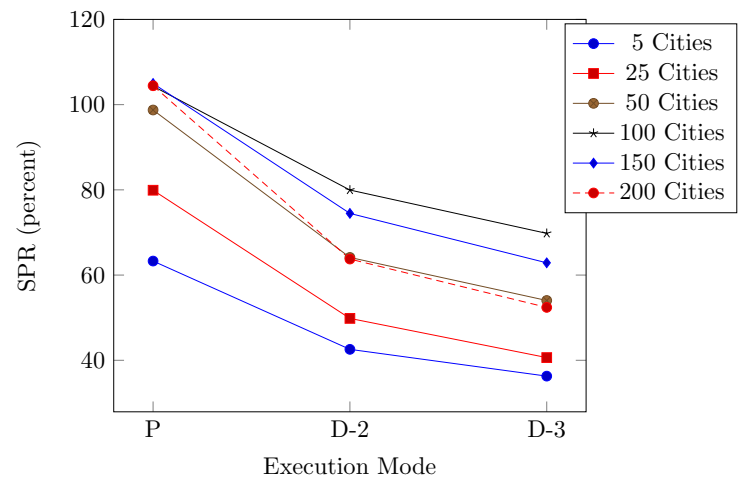

Figure 9: Execution Mode vs. Number of Cities for SPR (LKH) 


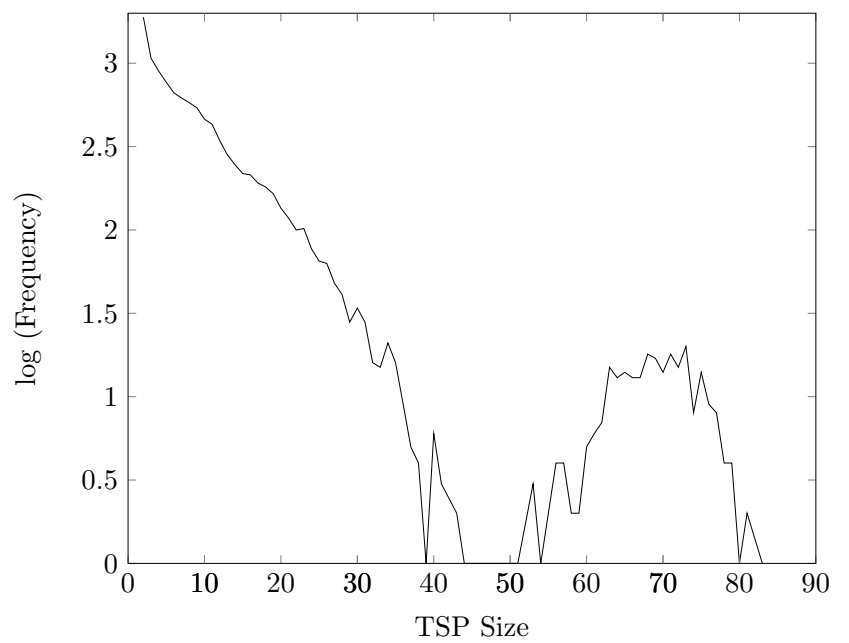

Figure 10: TSP Size Frequency for 10,967 TSPs (Three outliers, 162, 163, and 164 are omitted from the graph)

10,967 TSPs, the average number of cities visited per TSP is 10.12, and the largest TSP has 164 cities. The frequency of TSP sizes is shown in Figure 10. Because of variable TSP sizes, equal distribution of TSPs among identical machines may create overloaded machines and increase the total makespan. Therefore, we compare an equal distribution rule (EDR) against a well known task assignment rule, the longest processing time (LPT) rule [30]. In LPT, jobs (TSPs) are sorted by problem size (as a proxy for processing time, which we do not know yet) and assigned to the machine with the earliest end time so far. In worst case scenario, the algorithm achieves a makespan of $4 / 3-1 / 3(m) O P T$ where $m$ is the number of machines (i.e., LPT produces a makespan that is at most $33 \%$ worse than optimal assignment). We perform five replications for each experiment. Two important things affect the results of these runs: the order of the TSPs in EDR and the estimation of processing time by size of TSP. In some cases, a 40-city TSP can be much harder than a 41-city TSP. Also, a two machine EDR may perform close to optimal, whereas a three machine EDR can be quite suboptimal. The 3-way ANOVA in Table 6 shows that all main factors and the Machines*Solver interaction are significant. Other two-way interactions and the three way interaction are insignificant. The model explains $99.65 \%$ of the variability of the response data around its mean. Figure 11 shows the statistically significant effects - all three main effects and the two way interaction for Machines*Solver. The difference between the means of the TSP solvers is more pronounced than the other two main effects; LKH is nearly three times faster than Concorde. Obviously, increasing from two to three machines reduces the time needed. The 1.8 second difference between the means of EDR and LPT is statistically significant, showing the benefit of using LPT for unequal sizes of TSPs. The two way effect lines are not parallel, showing that Concorde benefits relatively more from using three machines than does LKH.

In order to show LPTs effectiveness, we create a more controlled experiment with generated TSPs. 
Table 5: ANOVA: Time versus Machines, Algorithm, Solver (Real Data)

\begin{tabular}{l|rr|r|r|r}
\hline Source & DF & Adj SS & Adj MS & F-Value & P-Value \\
\hline Machines & 1 & 912.11 & 912.11 & 996.58 & 0.000 \\
Solver & 1 & 7219.01 & 7219.01 & 7887.54 & 0.000 \\
Scheduling & 1 & 33.31 & 33.31 & 36.40 & 0.000 \\
Machines*Solver & 1 & 270.03 & 270.03 & 295.03 & 0.000 \\
Machines*Scheduling & 1 & 0.82 & 0.82 & 0.90 & 0.350 \\
Solver*Scheduling & 1 & 2.35 & 2.35 & 2.57 & 0.119 \\
Machines*Solver*Scheduling & 1 & 0.07 & 0.07 & 0.08 & 0.779 \\
\hline Error & 32 & 29.29 & 0.92 & & \\
\hline Total & 39 & 8467.01 & & & \\
\hline & $\mathbf{S}$ & R-sq & R-sq(adj) & R-sq(pred) & \\
\hline & 0.956683 & $99.65 \%$ & $99.58 \%$ & $99.46 \%$ & \\
\hline
\end{tabular}

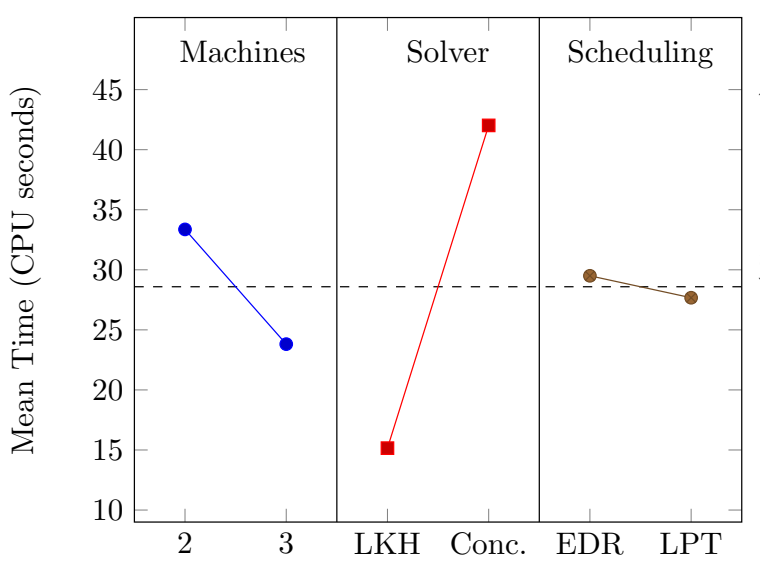

(a) Main Effects

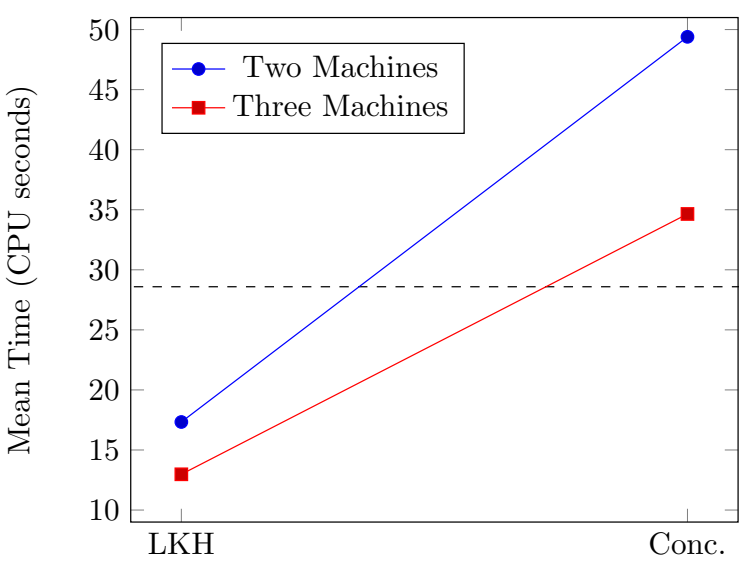

(b) Machines*Solver

Figure 11: Main Effects and Machines*Solver Interaction Plots for Time (Real Data) 
Table 6: ANOVA: Time versus Machines, Algorithm, Solver (Generated Data)

\begin{tabular}{l|rr|r|r|r}
\hline Source & DF & Adj SS & Adj MS & F-Value & P-Value \\
\hline Machines & 1 & 2555.9 & 2555.9 & 404.55 & 0.000 \\
Algorithm & 1 & 20244.7 & 20244.7 & 3204.36 & 0.000 \\
Scheduling & 1 & 403.5 & 403.5 & 63.87 & 0.000 \\
Machines*Solver & 1 & 718.9 & 718.9 & 113.79 & 0.000 \\
Machines*Scheduling & 1 & 8.2 & 8.2 & 1.29 & 0.264 \\
Solver*Scheduling & 1 & 173.1 & 173.1 & 27.40 & 0.000 \\
Machines*Solver*Scheduling & 1 & 36.2 & 36.2 & 5.73 & 0.023 \\
\hline Error & 32 & 29.29 & 0.92 & & \\
\hline Total & 39 & 8467.01 & & & \\
\hline & S & R-sq & R-sq(adj) & R-sq(pred) & \\
\hline & 0.956683 & $99.65 \%$ & $99.58 \%$ & $99.46 \%$ & \\
\hline
\end{tabular}

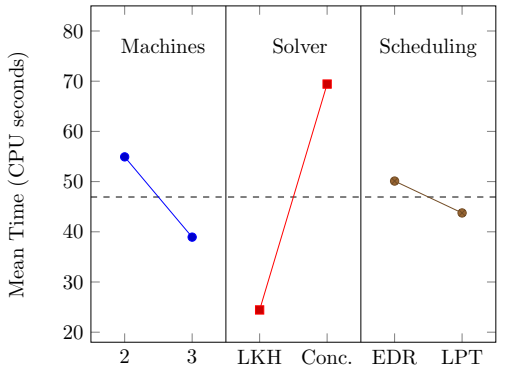

(a) Main Effects

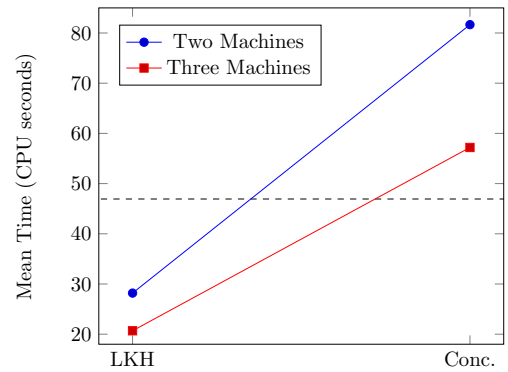

(b) Machines*Solver

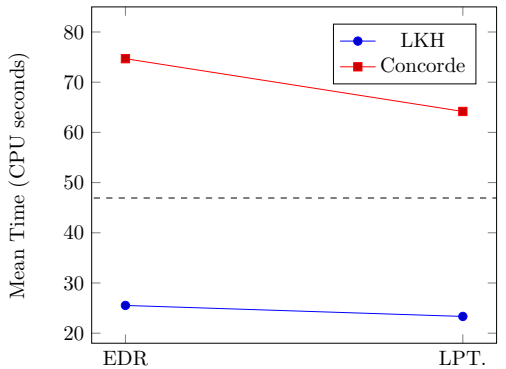

(c) Solver*Scheduling

Figure 12: Main Effects and Machines*Solver Interaction Plots for Time (Generated Data)

In this case, we create TSPs in the following order repeatedly for 3,840 times: size 51, size 11 , size 6 . In this way, we know that the EDR method will assign all 51-city TSPs to the first machine, all 11-city TSPs to the second machine, and all 6-city TSPs to the third machine. In this experiment, there are 11,520 TSPs and average TSP size is 22.67. All main effects, and two and three way interactions are statistically significant except for the Machines*Scheduling interaction. Figure 12 shows the three main effects and the significant two way interactions, Machines*Algorithm and Algorithm*Scheduling. The results are congruent with those from the real data. LKH is 2.84 times faster than Concorde on average. LPT finishes on the average 6.35 seconds earlier than EDR, again showing its benefit. The choice of the scheduling approach is more important when the TSP solver is Concorde because of the longer and non-uniform processing times for this exact method.

\section{Conclusions}

We presented how parallel computing techniques can significantly decrease the overall computational time and increase the CPU utilization for solving large batches of TSPs using simple and effective parallel class methods in C\#. Moreover, we showed our results for distributed and parallel computing methods 
with two and three slave machines. Using distributed computing techniques requires some background in $\mathrm{C} \#$ socket programming but simple examples can be found on the web [28].

Solving large batches of TSPs is the most computationally intensive step in many applications involving routing. Our results show that using C\# parallel class methods is a simple, effective and scalable way to parallelize solving large batches of TSPs. The programmer can write wrapper functions for Concorde and LKH, and implement "parallel for loops" to leverage the multi-core processors. However, distributed computing techniques only show their real benefits when the TSP instances have more than 50 cities so that the network and file read/write overhead is relatively negligible. Our results also show that for both real data and generated data, a scheduling algorithm like LPT performs better than a naïve method like EDR even though the method used for estimating processing times of TSPs is not very accurate (TSP size, in this case).

The Lin-Kernighan Heuristic (LKH) can be selected over the Concorde TSP Solver when optimality is desired but not required. In our results, LKH is $24.37,30.59,20.14$, and 21.91 times faster than Concorde on average in single, parallel, 2-computer distributed, and 3-computer distributed runs for solving 10,000 200-city TSPs, respectively. The average optimality gap is less than $0.34 \%$ per run.

\section{Acknowledgement}

This research was supported in part by the National Science Foundation under Grant CMMI-1200567. Any opinions, findings, and conclusions or recommendations expressed in this material are those of the authors and do not necessarily reflect the views of the National Science Foundation. The authors would like to thank Michael Robbins and Ataman Billor for data collection and testing the code.

\section{References}

[1] M. A. Ismail, S. H. Mirza, and T. Altaf, "A parallel and concurrent implementation of Lin-Kernighan heuristic (LKH2) for solving traveling salesman problem for multi-core processors using $\mathrm{SPC}^{3}$ programming model," International Journal of Advanced Computers Science and Applications, vol. 2, pp. 34-43, 2011.

[2] S. G. Ozden, A. E. Smith, and K. R. Gue, "Non-traditional warehouse design optimization and their effects on order picking operations." Working Paper, 2017.

[3] S. Kubota, T. Onoyama, K. Oyanagi, and S. Tsuruta, "Traveling salesman problem solving method fit for interactive repetitive simulation of large-scale distribution networks," in Systems, Man, and Cybernetics, 1999. IEEE SMC'99 Conference Proceedings. 1999 IEEE International Conference on, vol. 3, pp. 533-538, IEEE, 1999.

[4] Y. Sakurai, T. Onoyama, S. Kubota, Y. Nakamura, and S. Tsuruta, "A multi-world intelligent genetic algorithm to interactively optimize large-scale TSP," in 2006 IEEE International Conference on Information Reuse 8 Integration, pp. 248-255, IEEE, 2006.

[5] D. R. Kraay and P. T. Harker, "Case-based reasoning for repetitive combinatorial optimization problems, part II: numerical results," Journal of Heuristics, vol. 3, no. 1, pp. 25-42, 1997. 
[6] Y. Sakurai, K. Takada, N. Tsukamoto, T. Onoyama, R. Knauf, and S. Tsuruta, "A simple optimization method based on Backtrack and GA for delivery schedule," in 2011 IEEE Congress of Evolutionary Computation (CEC), pp. 2790-2797, IEEE, 2011.

[7] M. R. Garey and D. S. Johnson, "Computers and intractability: a guide to NP-completeness." WH Freeman New York, 1979 .

[8] K. Rocki and R. Suda, "High performance GPU accelerated local optimization in TSP," in Parallel and Distributed Processing Symposium Workshops \& PhD Forum (IPDPSW), 2013 IEEE 27th International, pp. 1788-1796, IEEE, 2013.

[9] M. Held and R. M. Karp, "A dynamic programming approach to sequencing problems," Journal of the Society for Industrial and Applied Mathematics, vol. 10, pp. 196-210, 1962.

[10] D. L. Applegate, R. E. Bixby, V. Chvátal, and W. J. Cook, The Traveling Salesman Problem: A Computational Study. Princeton University Press, 2007.

[11] W. Cook, In pursuit of the traveling salesman: mathematics at the limits of computation. Princeton University Press, 2014.

[12] "TSPLIB." http://comopt.ifi.uni-heidelberg.de/software/TSPLIB95/, 2013 [accessed 02.20.2016].

[13] S. Lin and B. W. Kernighan, "An effective heuristic algorithm for the traveling-salesman problem," Operations Research, vol. 21, no. 2, pp. 498-516, 1973.

[14] K. Helsgaun, "An effective implementation of the Lin-Kernighan traveling salesman heuristic," European Journal of Operational Research, vol. 126, pp. 106-130, 2000.

[15] S. Kirkpatrick, "Optimization by simulated annealing: Quantitative studies," Journal of Statistical Physics, vol. 34, no. 5-6, pp. 975-986, 1984.

[16] J. Grefenstette, R. Gopal, B. Rosmaita, and D. Van Gucht, "Genetic algorithms for the traveling salesman problem," in Proceedings of the First International Conference on Genetic Algorithms and their Applications, pp. 160-168, Lawrence Erlbaum, New Jersey (160-168), 1985.

[17] J. Knox, "Tabu search performance on the symmetric traveling salesman problem," Computers 6 Operations Research, vol. 21, no. 8, pp. 867-876, 1994.

[18] M. Dorigo and L. M. Gambardella, "Ant colonies for the travelling salesman problem," BioSystems, vol. 43, no. 2, pp. 73-81, 1997.

[19] H. R. Lourenço, O. C. Martin, and T. Stützle, Handbook of Metaheuristics, ch. Iterated Local Search, pp. 320-353. Boston, MA: Springer US, 2003.

[20] X. Shi, Y. Liang, H. Lee, C. Lu, and Q. Wang, "Particle swarm optimization-based algorithms for TSP and generalized TSP," Information Processing Letters, vol. 103, no. 5, pp. 169 - 176, 2007.

[21] L. Shi, S. Ólafsson, and N. Sun, "New parallel randomized algorithms for the traveling salesman problem," Computers \&6 Operations Research, vol. 26, no. 4, pp. 371-394, 1999.

[22] B. Angeniol, G. D. L. C. Vaubois, and J.-Y. Le Texier, "Self-organizing feature maps and the travelling salesman problem," Neural Networks, vol. 1, no. 4, pp. 289-293, 1988.

[23] M. Lazarova and P. Borovska, "Comparison of parallel metaheuristics for solving the TSP," in Proceedings of the 9th International Conference on Computer Systems and Technologies and Workshop for PhD Students in Computing, p. 17, ACM, 2008.

[24] MSDN, "Parallel loops." https://msdn.microsoft.com/en-us/library/ff963552.aspx, 2016 [accessed 02.20.2016].

[25] I. A. Aziz, N. Haron, M. Mehat, L. Jung, A. N. Mustapa, and E. Akhir, "Solving traveling salesman problem on cluster compute nodes," WSEAS Transactions on Computers, vol. 8, no. 6, pp. 1020-1029, 2009. 
[26] G. Cesari, "Divide and conquer strategies for parallel TSP heuristics," Computers \& Operations Research, vol. 23, no. 7, pp. 681-694, 1996.

[27] MSDN, "Parallel class." https://msdn.microsoft.com/en-us/library/system.threading.tasks.parallel (v=vs. 110) .aspx, 2016 [accessed 02.20.2016].

[28] MSDN, "Asynchronous client socket example." https://msdn.microsoft.com/en-us/library/bew39x2a\%28v=vs.110\% 29. aspx?f=255\\&MSPPError=-2147217396, 2016 [accessed 02.20.2016].

[29] S. Casey, "How to determine the effectiveness of hyper-threading technology with an application." https://software.intel.com/en-us/articles/how-to-determine-the-effectiveness-of-hyper-threading-technology-withan-application/, 2011 [accessed 06.13.2016].

[30] R. L. Graham, "Bounds on multiprocessing timing anomalies," SIAM Journal on Applied Mathematics, vol. 17, no. 2, pp. 416-429, 1969. 
Table A.7: Concorde Average Execution Times and Average Cost per Tour

\begin{tabular}{|c|c|c|c|c|c|c|}
\hline & \multicolumn{5}{|c|}{ Average Execution Time (seconds) } & \multirow[b]{2}{*}{ Avg. Cost } \\
\hline & \# of TSPs & Single & Parallel & Distributed 2 & Distributed 3 & \\
\hline \multirow[t]{4}{*}{5 Cities } & 10 & 0.27 & 0.07 & 0.05 & 0.04 & 740.20 \\
\hline & 100 & 2.73 & 0.59 & 0.35 & 0.27 & 782.50 \\
\hline & 1000 & 27.83 & 8.13 & 4.44 & 3.48 & 788.10 \\
\hline & 10000 & 281.20 & 84.19 & 52.98 & 44.00 & 786.20 \\
\hline \multirow[t]{4}{*}{25 Cities } & 10 & 0.66 & 0.14 & 0.11 & 0.11 & 1466.80 \\
\hline & 100 & 6.71 & 1.48 & 0.68 & 0.53 & 1490.90 \\
\hline & 1000 & 70.33 & 18.89 & 6.80 & 5.19 & 1499.60 \\
\hline & 10000 & 684.64 & 183.48 & 74.28 & 53.68 & 1499.80 \\
\hline \multirow{4}{*}{50 Cities } & 10 & 1.53 & 0.41 & 0.32 & 0.37 & 2215.50 \\
\hline & 100 & 16.33 & 4.47 & 2.33 & 2.63 & 2203.90 \\
\hline & 1000 & 180.18 & 38.91 & 17.99 & 13.35 & 2208.80 \\
\hline & 10000 & 1763.84 & 384.82 & 167.74 & 117.51 & 2208.00 \\
\hline \multirow[t]{4}{*}{100 Cities } & 10 & 6.21 & 1.44 & 1.32 & 1.20 & 2747.80 \\
\hline & 100 & 150.80 & 28.02 & 19.20 & 14.99 & 2826.10 \\
\hline & 1000 & 1484.11 & 224.42 & 124.68 & 88.17 & 2807.20 \\
\hline & 10000 & 14081.30 & 2070.64 & 1065.72 & 728.33 & 2807.40 \\
\hline \multirow[t]{4}{*}{150 Cities } & 10 & 21.25 & 14.84 & 20.66 & 15.85 & 3055.32 \\
\hline & 100 & 358.97 & 66.28 & 63.61 & 70.01 & 3055.05 \\
\hline & 1000 & 3882.53 & 580.35 & 351.87 & 269.67 & 3053.73 \\
\hline & 10000 & 39560.49 & 5492.05 & 3164.54 & 2238.90 & 3050.34 \\
\hline \multirow[t]{4}{*}{200 Cities } & 10 & 66.58 & 30.09 & 131.03 & 29.17 & 3148.05 \\
\hline & 100 & 743.70 & 143.93 & 309.28 & 139.23 & 3161.00 \\
\hline & 1000 & 7594.09 & 1173.47 & 719.45 & 799.00 & 3160.18 \\
\hline & 10000 & 74592.12 & 12502.08 & 6082.22 & 5193.40 & 3157.99 \\
\hline
\end{tabular}

\section{Appendix A. Computational Time}

Tables A.7 and A.8 show the average execution times and average tour costs of Concorde and LKH. In all experiments, we use a single cross aisle traditional warehouse layout. The warehouse can accomodate 1000 items and these items are randomly distributed throughout the storage locations. We generated 10, 100, 1000, and 10000 orders with 4, 24, 49, 99, 149, and 199 items, and we calculated the distance of each order using TSP solvers. Therefore the average cost for 5 cities and 10 \# of TSPs is the average distance of completing 10 orders where each order has 4 items. Since each order has to start and end at the depot, picking 4 items in a warehouse is a 5-city TSP. 
Table A.8: LKH Average Execution Times and Average Cost per Tour

\begin{tabular}{|c|c|c|c|c|c|c|}
\hline & \multicolumn{5}{|c|}{ Average Execution Time (seconds) } & \multirow[b]{2}{*}{ Avg. Cost } \\
\hline & \# of TSPs & Single & Parallel & Distributed 2 & Distributed 3 & \\
\hline \multirow[t]{4}{*}{5 Cities } & 10 & 0.11 & 0.04 & 0.03 & 0.02 & 740.20 \\
\hline & 100 & 1.13 & 0.30 & 0.22 & 0.16 & 782.50 \\
\hline & 1000 & 11.29 & 2.66 & 1.79 & 1.43 & 788.10 \\
\hline & 10000 & 114.15 & 28.29 & 24.40 & 20.84 & 786.20 \\
\hline \multirow[t]{4}{*}{25 Cities } & 10 & 0.21 & 0.05 & 0.05 & 0.04 & 1466.80 \\
\hline & 100 & 1.80 & 0.41 & 0.30 & 0.24 & 1490.90 \\
\hline & 1000 & 19.00 & 3.39 & 2.52 & 2.05 & 1499.60 \\
\hline & 10000 & 185.82 & 36.03 & 31.68 & 24.18 & 1499.80 \\
\hline \multirow[t]{4}{*}{50 Cities } & 10 & 0.65 & 0.16 & 0.14 & 0.12 & 2215.50 \\
\hline & 100 & 5.51 & 0.90 & 0.68 & 0.57 & 2204.10 \\
\hline & 1000 & 55.85 & 8.27 & 6.07 & 4.56 & 2209.00 \\
\hline & 10000 & 551.92 & 81.21 & 62.35 & 47.73 & 2208.20 \\
\hline \multirow[t]{4}{*}{100 Cities } & 10 & 2.05 & 0.50 & 0.46 & 0.44 & 2752.90 \\
\hline & 100 & 25.72 & 3.84 & 2.46 & 2.00 & 2829.20 \\
\hline & 1000 & 249.86 & 35.78 & 22.03 & 15.73 & 2809.80 \\
\hline & 10000 & 2514.98 & 348.26 & 208.26 & 150.25 & 2809.90 \\
\hline \multirow[t]{4}{*}{150 Cities } & 10 & 2.90 & 0.71 & 0.74 & 0.67 & 3064.88 \\
\hline & 100 & 31.64 & 4.83 & 2.46 & 2.69 & 3065.36 \\
\hline & 1000 & 331.69 & 46.91 & 30.73 & 23.22 & 3063.17 \\
\hline & 10000 & 3310.92 & 449.05 & 287.87 & 223.98 & 3060.00 \\
\hline \multirow[t]{4}{*}{200 Cities } & 10 & 3.67 & 1.01 & 1.06 & 1.02 & 3158.04 \\
\hline & 100 & 30.64 & 4.51 & 4.00 & 3.31 & 3169.81 \\
\hline & 1000 & 291.98 & 40.98 & 31.48 & 24.66 & 3170.03 \\
\hline & 10000 & 3060.64 & 408.72 & 302.00 & 236.98 & 3168.13 \\
\hline
\end{tabular}

